\title{
Pénétration de la lumière au niveau du sous-bois d'une forêt dense tropicale
}

\author{
D.Y. ALEXANDRE \\ O.R.S.T.O.M., B.P. V 51, Abidjan (Côte-d'Ivoire) (*)
}

\section{Résumé}

Nous proposons un modèle simple de la pénétration de la lumière diffuse à travers un couvert végétal dense aléatoire "sphérique», tenant compte de la dispersion (transmission et réflexion) de la lumière par les feuilles :

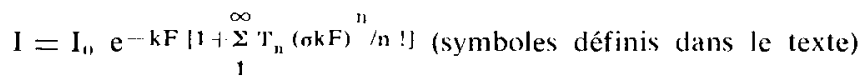

qui dans le cas du visible peut s'écrire :

$$
\mathbf{I}=\mathrm{I}_{4} \mathrm{e}^{-\mathrm{kF}(1-\mathrm{T} / 2)}
$$

et dans le cas général. pour les indices foliaires $(F)$ généralement rencontrés en forêt :

$$
\mathrm{I}=\mathbf{I}_{1}, \mathrm{e}^{-k F\left(1,1.4 \sigma^{\mathrm{T}}\right)}
$$

où $\sigma$ est un coefficient de dispersion que nous sommes amenés à introduire pour tenir compte du fait que la dispersion par les feuilles s'accompagne d'une modification cle la distribution angulaire des rayons.

Les valeurs moyennes de l'éclairement du sous-bois de la forêt dense équatoriale de Taï (Côtc-d'Ivoire) mesuré dans deux bandes spectrales $(0,4$ à $0,7 \mu$ avec le quantomètre et 0,4 ì 1,1 u avec le "pyranomètre " Lambcla) montrent que les hypothèses retenues traduisent, pour ce type de couvert, la réalité de façon acceptable.

Nous montrons également qu'en sous-bois, le rapport des éclairements dans deux bandes spectrales différemment transmises par les feuilles est pratiquement constant et ne permet donc pas d'estimer l'indice foliaire du peuplement. Le modèle montre que cette constance du rapport spectral s'explique par une variation de la valeur du coefficient $\sigma$ et que celui-ci doit inclure le pourcentage d'organes foliaires (indice foliaire sensu stricto) dans l'ensemble des éléments qui font obstacle à la pénétration de la lumière (indice surfacique).

Nous montrons enfin que malgré la différence de réponse specctrale, les classiques piles au sélénium (luxmètres) peuvent être utilisées à la place d'un quantomètre, à condition qu'elles soient pourvues d'une bonne correction angulaire.

$$
\text { Mots-clés : Modélisation - Eclairement - Forêt - Côte-d'toire }
$$

(") Adresse actuelle: Laboratoire d'Ecologie végétale, Université de Paris-Sud, bâtiment 43I, F 91405 Orsay. 


\section{Introduction}

L'étude des principaux mécanismes qui entrent en jeu au cours de la régénération naturelle de la forêt dense équatoriale de Côte-d'Ivoire nous a montré la nécessité d'une meilleure connaissance de l'éclairement du sous-bois de la forêt intacte. En effet, bien que les facteurs biotiques prennent, dans ces biocénoses, une importance particulière, il n'en demeure pas moins que le facteur lumière, sous ses différents aspects, reste primordial car, si l'essentiel de la régénération du couvert se fait à la faveur des trouées, c'est en sous-bois, pendant la phase obscure, que s'établit le potentiel floristique qui s'exprime dès que l'éclairement augmente (AlEXandre, $1979 \mathrm{c}$ et d) ; «Linsuffisance de la lumière est le plus important des facteurs microclimatiques à considérer en forêt dense, pour la régénération» (CHARTIER et al., 1973).

L'éclairement du sous-bois est, on le sait, très variable dans le temps et dans l'espace et il est indispensable, pour pouvoir le décrire, d'avoir une certaine compréhension théorique de la pénétration de la lumière solaire qui dépend, pour une part, de l'agencement dans l'espace des organes constitutifs de la forêt (structure) et, d'autre part, du rayonnement lumineux lui-même.

La modélisation est un des moyens les plus utilisés pour étudier la pénétration des rayonnements à travers les couverts. Un modèle traduit de façon synthétique un ensemble d'hypothèses comme, par exemple, le caractère aléatoire de la position et de l'inclinaison des feuilles dans le cas du couvert de la forêt dense tropicale. De la confrontation du modèle avec l'objet qu'il représente naissent des conclusions sur la validité des hypothèses, qui rendent possible une nouvelle étape d'élaboration du modèle. L'emploi de la modélisation est donc une démarche essentiellement itérative qui peut permettre, dans les cas favorables, dappréhender des facteurs qui échapperaient à d'autres moyens d'investigation.

Ainsi nous ne chercherons pas un modèle pour prédire avec précision telle ou telle quantité relative aux phénomènes radiatifs, tant il est vrai que pour une grandeur précise «la mesure directe serait souvent plus simple et plus rapide que celle des paramètres qui en permettent le calcul» (CowAN, 1968), mais bien un modèle qui permette danalyser une certaine cohérence globale tout en restant suffisamment simple pour ne pas nécessiter de gros moyens de calcul. La littérature abonde, il est vrai, en modèles variés traitant de divers aspects de la pénétration du rayonnement au sein de couverts végétaux, mais ceux que nous avons étudiés nous sont apparus ou inadéquats ou trop complexes pour notre propos.

Le modèle que nous présentons et que nous discutons ici est basé sur un maximum d'hypothèses simplificatrices (feuilles à distribution, orientation et inclinaison aléatoires, disposées en couches indépendantes) mais prend en compte la dispersion (transmission et réflexion) des rayons lumineux par les feuilles. Nous confronterons le modèle à la fois avec la forêt dense dite «climacique » qui est notre principal objet d'intérêt, mais également avec différentes formes de dégradation ou de reconstitution de cette forêt. 


\section{Rappel d'éléments théoriques : pénétration du rayonnement photosynthétiquement actif}

Tous les modèles de pénétration de la lumière dans un couvert s'expriment par une loi de Beer-Lambert de la forme :

$$
\mathrm{I}=\mathrm{I}_{\mathrm{o}} \mathrm{e}^{-\mathrm{kF}}
$$

qu'ils soient dérivés de la loi de Poisson, de lois binomiales positives ou négatives ou même de séries markoviennes (Nilson, 1971 ; Lemeur \& BL.AD, 1974).

La loi de Poisson rend compte du cas limite le plus simple, celui du couvert dont les couches sont indépendantes (aléatoires). C'est elle qui est utilisée le plus souvent et c'est souvent la seule utilisable, a priori, les autres demandant la connaissance d'autres paramètres que l'indice foliaire $F$, en particulier des paramètres d'associativité, de recouvrement préférentiel ou non...

Dans le visible (ou clomaine des radiations utiles à la photosynthèse $=\mathrm{PAR}$ ) où transmission et réflexion sont faibles, on considère souvent la feuille comme opaque, ce qui apporte une énorme simplification. Dans ce cas en effet, calculer l'éclairement relatif à un niveau défini par son indice foliaire $F$ cumulé à partir du sommet du couvert, revient à calculer la probabilité d'une absence de rencontre entre rayon lumineux et feuilles (ANDERSON, 1966).

Pour un couvert dont les couches sont indépendantes, cette probabilité sera bien évidemment donnée par le terme zéro de la loi de Poisson. On aura :

$$
\mathrm{I}=\mathrm{I}_{\mathrm{o} \alpha} \mathrm{e}^{-\mathrm{kF} / \cos \alpha}
$$

où $F$ est l'indice foliaire jusqu'au niveau de mesure, $\alpha$ l'angle du rayon lumineux avec la verticale et $\mathrm{kF}$ la surface efficace des feuilles, c'est-à-dire leur projection dans le plan orthogonal au rayon Iumineux $(\mathrm{kF} / \cos \alpha$ représente la projection dans le plan horizontal de cette projection, $\mathrm{k}$ dépend de la structure angulaire des feuilles).

Dans le cas d'un couvert sphérique défini par une équiprobabilité des normales aux feuilles, $k$ prend la valeur $1 / 2$ (Cowan, 1961). Si maintenant l'on considère indépendamment un azimut et une inclinaison aléatoire, on obtient une autre distribution "entièrement aléatoire », utilisée par CHARTIER (1966) pour son couvert dense hétérogène, où $\mathrm{k}=2 / \pi$. done :

La loi de Beer-Lambert pour un couvert sphérique de feuilles opaques est

$$
\mathrm{I}=\mathrm{I}_{\mathrm{or}} \mathrm{e}^{-\mathrm{F} / 2 \cos \alpha}
$$

$\mathrm{k}$ augmente quand l'angle d'incidence augmente, c'est-à-dire que les rayons obliques sont plus rapidement captés que les rayons verticaux; en conséquence, ainsi que l'ont montré ANDerson (1966) et Cowan (1968), la relation (3) implique que pour un rayonnement diffus, la part relative du rayonnement provenant du zénith croît au fur et à mesure que l'on pénètre dans le couvert ou, en d'autres termes, que $\mathrm{F}$ augmente. Sans que la structure du feuillage varie, le $\mathrm{k}$ des couches profondes est donc plus petit que celui des couches supérieures et à l'échelle du couvert entier, 
$\mathrm{K}$ pour le rayonnement diffus (1) diminue quand $\mathrm{F}$ augmente. Si l'on considère que : * pour un ciel uniforme (UOC) la lumière incidente sur une surface horizontale provenant d'un angle $\beta$ est équivalente à la projection d'une sphère unité (WaLSH, 1961 ), $\mathbf{I}_{\beta}=\sin 2 \beta$ ( $\beta$ est l'élévation) » (d'après ANDERSON, loc. cit.), on peut calculer $\mathrm{K}$ et $\mathrm{KF}$ pour différentes valeurs de $\mathrm{F}$. C'est ainsi qu'on a obtenu la courbe UOC de la fig. 1 . A titre d'exemple, $K=0,76$ pour $F=2$ et $K=0,65$ pour $F=8,3$ (cette valeur est une valeur probable de l'indice foliaire en forêt tropicale : AlEXANDRE, 1979 b).

En pratique, la luminance du ciel varie avec l'élévation $\beta$. On obtient une représentation proche de la réalité avec le «ciel couvert standard» (SOC) dont la luminance $\mathrm{L}_{90}$ au zénith est trois fois plus grande qu'à l'horizon et obéit à la relation empirique :

$$
\mathbf{L}_{\beta}=2 / 3 \mathbf{L}_{\mathbf{9}(1)}(1+2 \sin \beta) \quad \text { (MOON \& SPENCER, 1942) }
$$

ce qui conduit à un éclairement du plan :

$$
\mathrm{I}_{\beta}=2 / 3\left(\sin \beta+2 \sin ^{2} \beta\right) \cos \beta \quad \text { (ANDERSON, loc. cit.) }
$$

avec lequel on peut également calculer $\mathrm{K}$ et KF (SOC de la fig. 1). A titre de comparaison on obtient maintenant pour $F=2, K=0,71$ et pour $F=8,3, K=0,63$. Les valeurs de $\mathrm{K}$ pour un ciel standard sont donc légèrement plus petites que pour un ciel uniforme, l'écart diminue quand $\mathrm{F}$ augmente. En fait, in natura, on constate que les feuilles des couverts denses ont une tendance à la planophilie (port horizontal) qui augmente manifestement de la voûte vers le sous-bois; il est donc très peu probable qu'on constate effectivement une baisse du coefficient $K$ en fonction de l'indice foliaire sur un couvert réel.

L'étude d'autres phénomènes que la photosynthèse, et en particulier l'étude de la germination, conduit à considérer dautres parties du spectre lumineux que le visible (PAR : $0,4-0,7 \mu$ ) seul. Or si dans le visible les feuilles sont effectivement, en première approximation, presque opaques, dans l'infra-rouge et plus particulièrement dans l'infra-rouge très proche (également appelé rouge sombre), elles n'absorbent au contraire pratiquement pas. Il faut donc tenir compte impérativement de la réflexion et de la transmission et c'est ici qu'on se rend compte que les modèles deviennent très complexes et sont souvent loin de la réalité ; ils sont le plus souvent inutilisables en ce qui nous concerne.

\section{Proposition d'un modèle simple}

On conçoit aisément que le rayonnement au niveau du sol de la forêt dense est la somme des rayonnements qui y parviennent après avoir rencontré $0,1,2, \ldots$ feuilles. Dans une situation où le premier terme de la loi de Poisson $\left(\mathrm{P}_{o}=\mathrm{e}^{-\mathrm{kF}}\right)$

(1) Le symbole $\mathrm{K}$ (lettre majuscule) désigne le coefficient d'absorption de l'ensemble du couvert pour le rayonnement diffus, il intègre tous les paramètres du couvert et du rayonnement à l'exception de l'indice foliaire $F$. 
peut servir à calculer la probabilité de rencontrer zéro feuille, les termes suivants $\mathrm{P}(\mathrm{n})=\mathrm{e}^{-\mathrm{kF}} \cdot(\mathrm{kF})^{\mathrm{n} / \mathrm{n}}$ ! peuvent également servir à calculer la probabilité de rencontrer 1, 2, 3... feuilles (Niıson, 1971).

Mais la transmission et la réflexion affectent la distribution spatiale des rayons lumineux, ce qui modifie leurs trajets et donc leur probabilité d'arriver au niveau de mesure. On est donc amené à introduire un coefficient correcteur $(\sigma)$ pour rendre compte d'une diffusion.

Si la dispersion affecte pareillement tous les rayons (isotropie), on a :

$$
P(n)=\frac{(\sigma k F)^{n}}{n !} e^{-k F}
$$

où $P(n)$ est la fréquence relative des rayons qui ont touché $n$ feuilles.

Rappelons bien qu'il s’agit de l'étude d'un cas limite, le plus simple, celui du couvert sphérique, pour un ciel uniforme, pour lequel on admettra avec BonHOMme \& Varlet-Grancher (1977) que la somme du rayonnement transmis et du rayonnement réfléchi est isotrope.

\subsection{Eléments de justification de la relation précédente}

La probabilité d'arriver au niveau $F$ après avoir touché 1 feuille est égale à la probabilité de toucher 1 feuille avant le niveau $\mathrm{f}$ puis de n'en plus toucher :

$$
P_{1}=k f e^{-k f} \cdot e^{-k(F-f)}=k f e^{-k F}
$$

f prenant, par hypothèse, n“importe quelle valeur entre $O$ et $F$ avec une égale probabilité puisque nous admettons la loi de Poisson (cf. Nilson, 1971), il vient naturellement :

$$
P_{1}=1 / 2 \mathrm{kF} \mathrm{e}^{-k F} \quad(\overline{\mathrm{f}}=1 / 2 \mathrm{~F})
$$

Dans ce cas, $\sigma=1 / 2$. sera :

L'éclairement relatif provenant des rayons transmis et réfléchis par 1 feuille

$$
\delta_{1}=1 / 2\left(1 / 2 k F e^{-k F} \cdot T_{1}+1 / 2 k F e^{-k F} R_{1}\right)
$$

puisqu'on ne considère que la moitié du rayonnement, celle qui descend. Avec l'hypothèse $T_{1}=R_{1}$, généralement admise en première approximation, il vient :

$$
\delta_{1}=1 / 2 \mathrm{~T}_{1} k F e^{-k F}
$$

On calcule de mêne une probabilité de deux chocs de la forme :

$$
P_{.2}=(1 / 2)^{2}(k F)^{2 / 2} \quad e^{-k F}
$$




\subsection{Généralisation et application au rayonnement diffus au sol}

Au total, si l'on admet l'extension aux termes suivants, l'éclairement diffus relatif (EDR ou $\delta)$ est égal à :

$$
\mathrm{EDR}=\mathrm{e}^{-\mathrm{kF}}\left(1+\sum_{1}^{\infty} \mathrm{T}_{\mathrm{n}} \frac{(\sigma \mathrm{kF})^{\mathrm{n}}}{\mathrm{n} !}\right)
$$

où $T_{n}$ est le coefficient de transmission d'un nombre $\mathrm{n}$ de feuilles.

Dans le visible où $\mathrm{T}$ est petit et $\mathrm{T} \sigma \mathrm{kF}$ petit devant 1 , on aura en utilisant l'approximation $\log (1+\varepsilon) \simeq \varepsilon$ :

$$
\operatorname{EDR}_{\mathrm{vis}}=\mathrm{e}^{-(1-\sigma \mathrm{T}) \mathrm{kF}}=\mathrm{e}^{-\mathrm{k}^{\prime} \mathrm{F}}
$$

Graphiquement on obtient une solution approchée générale (quels que soient $\mathrm{T}$ (qui dépend de la bande spectrale), $\sigma$ et $\mathbf{k}$ ) :

$$
\mathrm{I} / \mathrm{I}_{\mathrm{o}}=\mathrm{e}^{-(1-1,4 \sigma \mathrm{T}) \mathrm{kF}}
$$

Quelques auteurs comme Kasanaga \& Monsi (1954), Chartier (1966) ou Monteith (1969) donnent une expression analogue :

$$
\mathbf{I} / \mathbf{I}_{\mathbf{o}}=\mathrm{e}^{-(1-\mathrm{T}) \mathrm{kF}}
$$

expression qui diffère de celle à laquelle nous arrivons par un coefficient 0,5 (équation 6) ou 0,7 (équation 6').

Notre modèle prévoit donc, dans le cas où $\sigma=1 / 2$, une part de transmission, due à la dispersion par les feuilles, plus petite que les modèles cités; la différence est négligeable dans le visible mais peut être très importante dans le proche infrarouge. Remarquons quelques aspects de la transmission qui, sans toujours expliquer la différence des formulations, ne nous semblent pas clairement considérés dans les travaux cités :

- d'une part : les réflexions multiples qui accompagnent la traversée du couvert, tendant à augmenter faiblement la transparence du couvert ;

- d'autre part : la dispersion des rayons qui accompagne à la fois la transmission et la réflexion et conduit, selon ce qui a été dit plus haut sur le rôle de l'inclinaison, à une diminution ou une augmentation de la transparence suivant limportance de l'inclinaison du rayonnement incident;

- et enfin : l'obliquité des rayons par rapport aux feuilles : le coefficient de transmission généralement utilisé est mesuré avec un rayon incident normal à la feuille ce qui surestime la transparence moyenne des feuilles et donc celle du couvert. $\mathrm{Si}$ l'on admet que l'absorption de la lumière est proportionnelle à l'épaisseur de feuille traversée, la transparence pour un rayon oblique $\mathrm{T}^{\prime}=\mathrm{T}_{0} \cos \beta$, où $\beta$ est l'angle entre le rayon et la normale à la feuille et $T_{0}$ la transparence pour un rayon normal. Dans le cas d'une distribution sphérique, $T=T_{0} / 2$. Ce mode de calcul n'est admis-

(*) Comme l'a montré YODA (1974) et comme nous l'avons également mesuré, les valeurs de l'EDR sont distribuées selon une loi log-normale, ce qui impliquerait une distribution normale de la grandeur F qui serait ainsi distribuée comme la majorité des variables biométriques. 
sible que pour le visible où la transmissivité dépend essentiellement de la teneur en chlorophylle tandis que dans linfra-rouge proche elle est surtout fonction de la structure de la feuille (GaUsman et al., 1970).

\subsection{Rôle de l'estimation du coefficient $T$}

Ainsi que nous le verrons plus loin, nous mesurons la transparence en lumière diffuse, ce qui simplifie la pratique mais peut ne pas rendre compte de façon complète de la réalité complexe.

On aura noté que nous écrivons $T_{n}$ (transparence de $n$ feuilles) et non $T^{\prime \prime}$ transmission d'une feuille à la puissance $n$. C'est qu'en effet les coefficients de transmission des feuilles successives ne seraient constants que s'ils étaient mesurés dans une bande spectrale étroite. Dans une bande spectrale plus ou moins large, le coefficient de transmission individuel des feuilles successives augmente du fait de la disparition progressive des bandes les plus absorbées.

Pour mesurer $T_{n}$, nous avons empilé les feuilles, immédiatement après récolte, sur les mêmes capteurs (capteurs Lambda) que ceux utilisés en forêt, et avons procédé à des mesures par temps couvert et dans un espace largement ouvert. Ainsi de cette façon $T_{u}$ intègre directement la bande spectrale incidente et ses modifications compte tenu des réflexions aller et retour. On peut montrer simplement que :

$$
T(n+1) / T(n)=T_{m}+T_{m}^{3}+2 T_{m}^{5}+5 T_{m}^{7}+\ldots
$$

où $T_{m}$ est la transmission propre d'une feuille dans la bande de transmission maximale si n est suffisamment élevé.

Ces deux facteurs (rôle du spectre et réflexions multiples) expliquent les mesures de Kasanaga \& Monsi (1954).

Le tableau I donne $T_{n}$ p. 100 moyen pour 24 espèces forestières fréquentes de la forêt du Banco (Turraeanthus africana, Dacryodes klaineana, Strombosia glaucescens, ...) (cf. Huttel, 1975).

\section{TABLEAU 1}

Pourcentages moyens de transinission en lumière diffuse de feuilles empilées des espèces les plus fréquentes de la forêt du Banco.

Mean diffuse light transmission factor of piled leaves of some of the commonest tree species in Banco-forest.

\begin{tabular}{|c|c|c|c|}
\hline & Visible 0,4 à 0,7 & $\begin{array}{c}\text { Visible }+ \text { IR proche } \\
0,4 \dot{\mathbf{a}} 1,1\end{array}$ & $\begin{array}{c}\text { IR proche } \\
790 \mathrm{~nm} \pm 75\end{array}$ \\
\hline 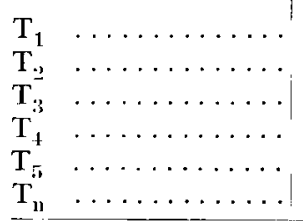 & $\begin{array}{l}4,23 \pm 0,71 \\
0,50 \pm 0,15\end{array}$ & $\begin{array}{c}37,88 \pm 1,49 \\
23,71 \pm 1,30 \\
16,35 \pm 1,17 \\
11,24 \pm 1,32 \\
8,74 \pm 0,89 \\
0,75 T_{n-1}\end{array}$ & $\begin{array}{l}58,14 \pm 1,88 \\
37,42 \pm 2,26 \\
26,14 \pm 1,98 \\
19,07 \pm 2,07 \\
13,94 \pm 1,85\end{array}$ \\
\hline
\end{tabular}


Quelques remarques peuvent être apportées à propos de ces valeurs :

- La transmission dune feuille dans l'infra-rouge très proche est très élevée, supérieure à $0,5(0,58)$. Cette valeur implique l'inégalité entre réflexion et transmission pour cette longueur d'onde.

Certaines espèces de climat tempéré ont également un coefficient de transmission dans linfra-rouge très élevé. Ainsi, à $800 \mathrm{~nm}$, le coefficient de transmission du colza (Szeicz, 1974), égal à celui du maïs (Gaussman, 1970), est de 0,53, celui du bananier, également selon Gausman, est de 0,52. L'effet de l'angle d'incidence est très faible à cette longueur d'onde.

- La valeur du coefficient de transmission obtenue pour la bande spectrale 0,4 à $1,1 \mu$ (vis. + proche infra-rouge) est également très élevée $(0,38)$. On remarque que cette valeur est supérieure à la moyenne des coefficients de transmission obtenus dans les bandes visibles $(0,4$ à $0,7 \mu, T=0,04)$ et proche infra-rouge $(0,79 \pm 0,075 \mu$, $\mathrm{T}=0,58$ ). Si lon tient compte de la part de rayonnement solaire représentée par chacune des deux bandes (environ 50 p. 100 de l'énergie dans le visible et 30 p. 100 dans le proche infra-rouge), la valeur moyenne pondérée que l'on obtient s'écarte encore davantage de la moyenne observée. Ceci s'explique par la courbe de sensibilité spectrale du capteur (cf, annexe n" 2) qui s'élève considérablement avec la longueur d'onde.

Quelques mesures effectuées en forêt de Taï vont maintenant montrer la validité ou l'intérêt du modèle.

\section{Etude du modèle}

\subsection{Utilisution de mesures de l'éclairement diffus relatif moyen du sous-hois de la forêt de Taï}

Une série de mesures systématiques de l'éclairement diffus relatif au niveau du sous-bois de la forêt de Taï a donné une moyenne arithmétique de 4,510-3 avec le quantomètre Lambda $(0,4-0,7)$ et $1,810^{-2}$ avec le «pyranomètre » $(0,4-1,1 \mu)$. Les mesures ont été faites en janvier, à une époque où les feuilles avaient peut-être commencé à tomber (Al.EXANDRE, 1979 a).

Dans une forêt dense thaïlandaise, également en saison sèche, YoDA trouve une valeur d'EDR très voisine : 4,1 10-3.

Dans les deux cas, les valeurs individuelles sont distribuées selon les lois lognormales, ce qui justifierait l'emploi de la moyenne géométrique et donnerait un chiffre légèrement plus petit pour l'EDR (soit un $k$ plus grand).

La mesure dans le visible permet d'utiliser la relation simplifiée (6) et de calculer le produit $\mathrm{kF}$ :

$$
k F=-\frac{\log \operatorname{EDR}_{\mathrm{vis}}}{1-\mathrm{T} / 2}=5,52(\mathrm{~T} \sim 4,23 \text { p. 100) }
$$


Cette valeur de $\mathrm{kF}$ peut être placée dans l'équation générale avec les diverses valeurs mesurées (cf. tableau) des coefficients de transmission pour la bande $0,4-1,1 \mu$. On a :

$$
\begin{aligned}
\mathrm{I} / \mathrm{I}_{\mathrm{o}}(0,4-1,1)= & \mathrm{e}^{-5,52}\left(1+0,379 \cdot 5,52 / 2+0,237 \cdot 1 / 2 \cdot(5,52 / 2)^{2}\right. \\
& +0,164 \cdot 1 / 6 \cdot(5,52 / 2)^{3}+\ldots
\end{aligned}
$$

(On remarque que les termes successifs du développement suivent approximativement une raison $1 / 2$, ce qui permet de donner à la somme des $\mathrm{n}$ derniers termes la valeur du terme $n-1$.)

Soit, finalement :

$$
\mathrm{I} / \mathrm{I}_{0}(0,4-1,1)=0,0161 \quad(\text { pour } \mathrm{n}-1=5)
$$

valeur très proche de la valeur observée : 0,018 .

Nous venons de montrer que la densité optique du couvert 5,40 [c'est-à-dire - $\log \left(I / I_{0}\right)$ avec $\left.I / I_{0}=0,045\right]$, serait théoriquement, si les feuilles étaient opaques, de 5,52 ; cette valeur correspond, pour un couvert sphérique et un ciel standard, à un indice foliaire de 8,9 (cf. fig. 1). Si l'on considère que l'indice foliaire d'une forêt tropicale peut atteindre au maximum la valeur de 8,2 (AlEXANDrE, 1979 b) et que la première valeur calculée $(8,9)$ s'applique à l'ensemble des objets qui font écran à la lumière (feuilles, branches, troncs...) tandis que la seconde ne considère que les feuilles, les deux valeurs peuvent être considérées comme en bon accord, et l'ensemble des résultats comme cohérent.

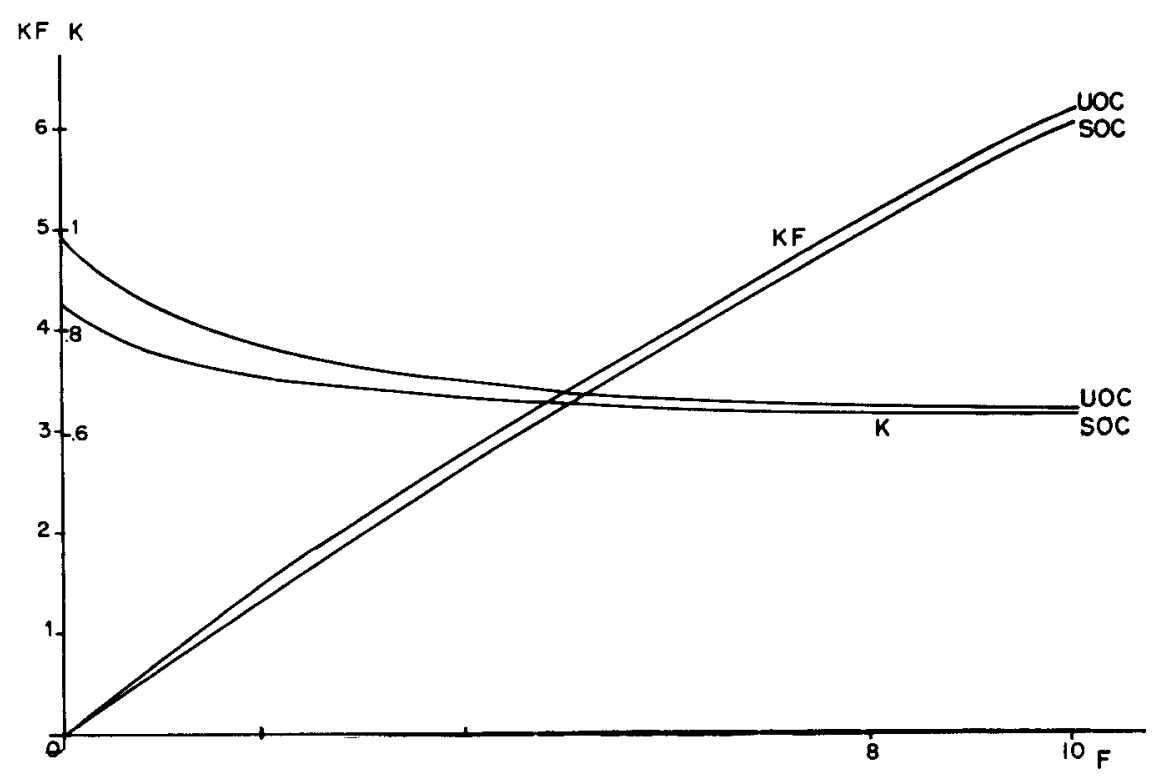

FIG. 1

Valeurs calculées du coefficient $K$ et du produit $K F$ pour un couvert sphérique et un ciel uniforme (UOC) et standard $(S O C)$ en fonction de l'indice foliaire $F$.

Computed values of the penetration factor $K$ and of the product $K F$ for an uniform overcast sky (UOC) or a standard overcast sky (SOC) for differing LAI $(F)$ in the case of a spherical stand. 
Nous pensons donc que notre modèle peut être utilisé dans l'état. Par exemple, pour comparer des forêts de structure analogue mais de densité différente, il permettra de calculer un indice foliaire à partir de la valeur de l'EDR en considérant le coefficient $K$ constant. Il pourra surtout être utile pour prévoir l'éclairement relatif dans des bandes spectrales particulières; il suffit pour cela de pouvoir mesurer ou estimer les coefficients de transmission des feuilles dans ces longueurs d'onde particulières. Ainsi pour la bande IR proche (tableau 1), on calcule un rayonnement relatif de 0,023 , plus de 5 fois la transparence dans le visible, ce que nous n'avons pas vérifié, mais nous verrons plus loin et plus en détail un exemple concret d'application avec les mesures au luxmètre.

Ce résultat positif nous a conduit à effectuer une deuxième série de mesures.

\subsection{Utilisation de mesures de la qualité spectrale de la lumière transmise par temps clair}

Après avoir vérifié que la moyenne des mesures de transmission s'accorde avec le modèle, nous allons maintenant considérer une par une les valeurs d'une nouvelle série de mesures effectuées cette fois par très beau temps, en dehors des taches de soleil, dans les deux mêmes bandes spectrales que précédemment $(0,4$ à 0,7 et 0,4 à $1,1 \mu)$. Une première série de mesures a été effectuée autour de midi solaire dans le sous-bois de parcelles intactes de la forêt de Taï et sous recrû de bord de route ; une deuxième série le soir, sous des formations de recrû, diverses, peu denses.

Sur la figure 2, on a porté, en coordonnées logarithmiques, les valeurs du rapport $R\left(R=i_{1} \cdot i_{02} / i_{2} \cdot i_{01}\right)$ des éclairements relatifs mesurés dans les deux bandes spectrales en fonction de l'éclairement relatif dans le visible. Malgré une certaine dispersion, qui peut n'être due qu'à la difficulté toujours grande de mesurer l'éclairement en dehors du couvert, une corrélation apparaît clairement.

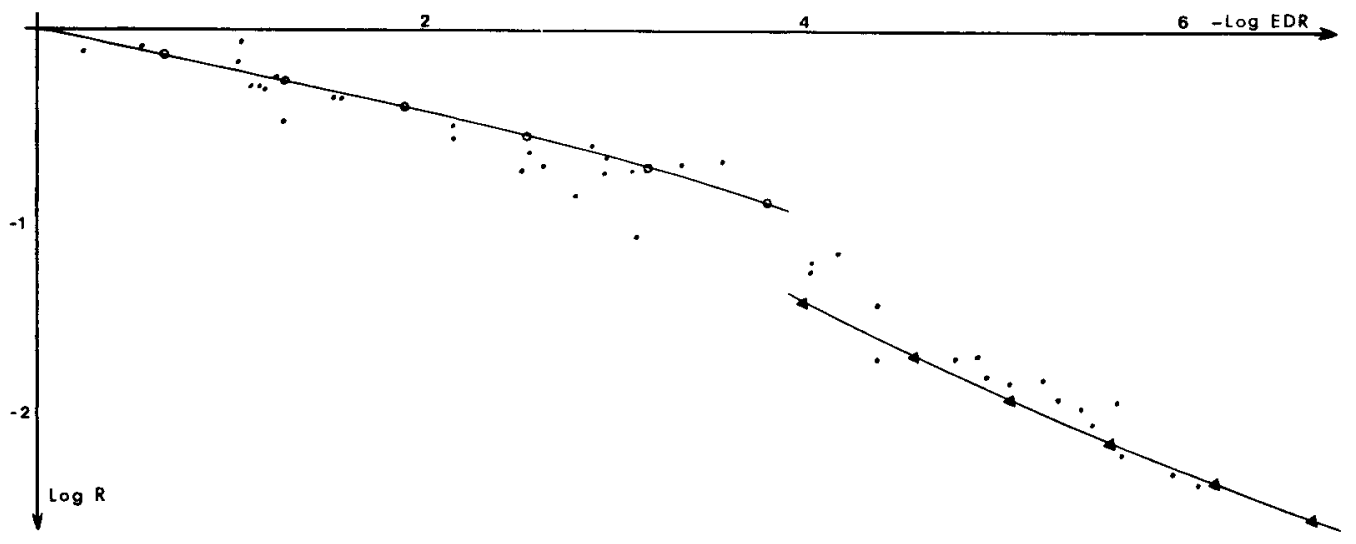

Fig. 2

Valeurs observées (points) et calculées du rapport $R$ en fonction de l'EDR (exprimés en logarithmes) pour un ciel couvert : courbe de gauche (ronds) et pour un ciel clair à midi : courbe de droite (triangles). Observed (dots) and calculated values of the ratio $R$ in relation with the relative light for an overcast sky (left, circles) and a clear day at noon (right, triangles). 
Il est intéressant de comparer les valeurs expérimentales avec les valeurs calculées à partir du modèle. Mais le modèle a été établi pour un ciel uniforme et ne s'applique donc pas à une période ensoleillée. Cependant, le soir, la valeur élevée du terme $1 / \cos \alpha$ autorise à négliger le rayonnement direct : le ciel se comporte vis-à-vis du couvert comme un ciel uniforme.

La dispersion élevée des points n'empêche pas de constater le bon accord entre les points expérimentaux et la courbe théorique obtenue à partir de quelques valeurs calculées.

Autour de midi, contrairement à ce qui se passe le soir, la pénétration dı direct est importante et il convient donc d'adapter le modèle. La transformation rigoureuse serait très complexe et conduirait à des expressions peu commodes. On se contentera donc d'une transformation approximative.

On considérera que le rayonnement transmis est la somme du rayonnement diffus selon le modèle et du rayonnement direct transmis selon le même modèle en prenant la valeur de $\mathrm{K}$ fonction de l'inclinaison $\alpha$ du rayonnement solaire direct (cf. relation 3). On se place, pour les mesures, en dehors des taches de soleil, on supprimera donc le premier terme de la série de Poisson, terme qui correspond à zéro rencontre, c'est-à-dire aux taches de soleil. Si l'on note par rd et rs les fractions par rapport au rayonnement global du diffus et du direct $(\mathrm{rd}+\mathrm{rs}=1)$, il vient :

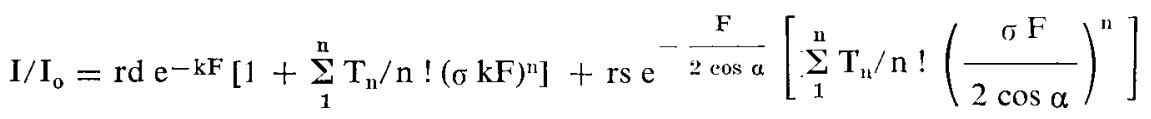

et dans notre cas, avec $\alpha \sim 0$ :

$$
I / I_{o}=\operatorname{rde} e^{-k F}\left[1+\sum_{1}^{n} T_{n} / n !(1 / 2 k F)^{n}\right]+\operatorname{rs~} e^{-F / 2}\left[\sum_{1}^{n} T_{n} / n !(F / 4)^{n}\right]
$$

Cette relation conduit (fig. 2), pour $\mathrm{rd}=1 / 3$, valeur rencontrée en Côte-d'Ivoire par très beau temps (MonTeny, com. pers.) et pour les valeurs de $T_{11}$ données précédemment (tableau 1), à la courbe théorique (étoiles). La similitude entre valeurs théoriques et expérimentales est, pour cette série de mesures, très satisfaisante. Pour un type de temps donné et un couvert donné, il y a bien un rapport étroit entre l'EDR et le rapport spectral $\mathrm{R}$ et l'on peut écrire : $\log \mathrm{EDR} / \log \mathrm{R}=$ cste.

L'indice foliaire est une caractéristique de grand intérêt pour un peuplement végétal mais sa mesure directe n'est pas envisageable pratiquement dans le cas d'une forêt tropicale.

Cet indice peut être déduit de la mesure de l'EDR en appliquant la loi de Beer-Lambert, $K$ étant estimé sur des bases théoriques comme nous l'avons vu précédemment, ou déduit d'observations isolées, et généralisé. Mais la mesure de l'EDR nécessite une mesure de référence $\left(I_{0}\right)$ au-dessus du couvert, très difficile à obtenir précisément dans le cas d'une haute forêt. La mesure de l'éclairement du sous-bois dans deux bandes spectrales différemment transmises devrait permettre, sur les mêmes bases théoriques, de calculer $\mathbf{R}$ sans avoir à effectuer de mesures au-dessus du couvert, donc plus facilement qu'à partir de l'EDR. 
Pour deux bandes spectrales 1 et 2 , on peut en effet déduire de $\left(6^{\prime}\right)$ avec $R=i_{1} \cdot i_{02} / i_{2} \cdot i_{01}$ (cf. supra) :

$$
F=\log R / 1,4 \sigma K\left(T_{1}-T_{2}\right)
$$

La technique a d'ailleurs été déjà utilisée par Odum, Copeland \& Brown (1963), à Puerto-Rico, semble-t-il avec succès. Soulignons que ces auteurs font leurs mesures au sein d'une seule et même forêt, sans tenter d'utiliser leur méthode pour comparer des forêts de structure a priori différentes.

Nous allons voir précisément que la technique n'est pas directement généralisable en raison des modifications a priori non quantifiables de la structure des couverts.

\subsection{Mesures de la transmissivité du couvert} au cours de la reconstitution forestière : essai de quantification de l'évolution structurale

Dans le but initial de mesurer l'évolution de l'indice foliaire au cours de la reconstitution, nous avons mesuré à laide du quantomètre, sensible de 0,4 à 0,7 , et du "pyranomètre », sensible de 0,4 à 1,1 , l'éclairement sous des recrûs forestiers homogènes d'âges différents.

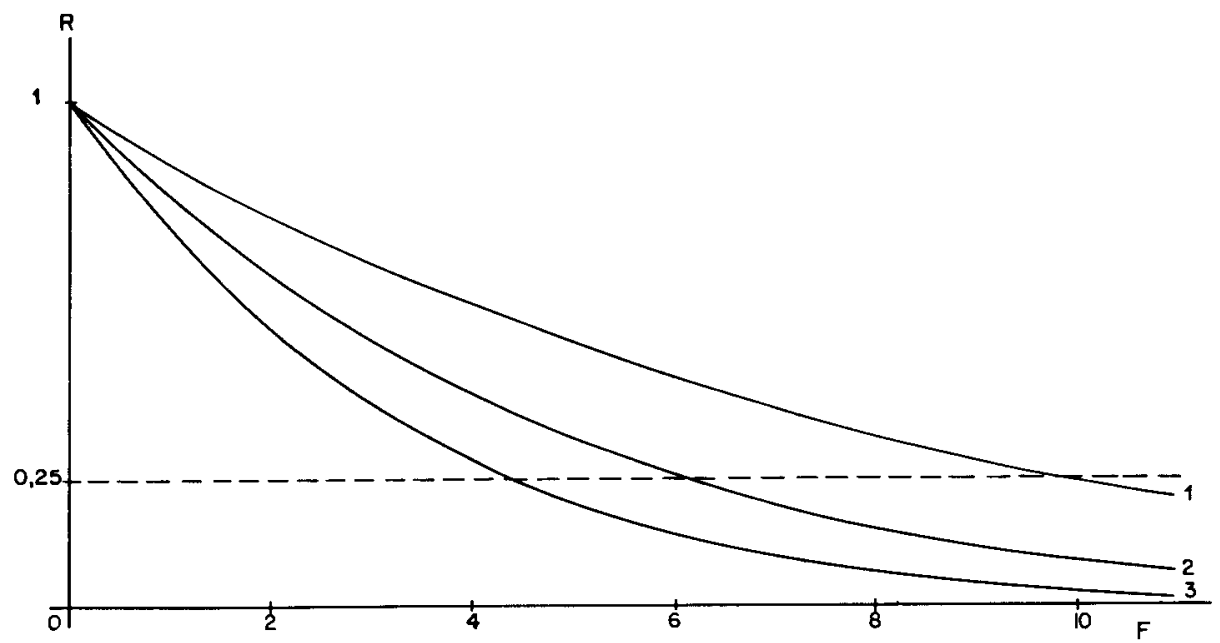

FIG. 3

Evolution du rapport $R$ en fonction de l'indice foliaire $F$ pour différentes valeurs du coefficient $\sigma$.

Variations of the ratio $R$ with the LAI (F) for $\sigma=.5, .75$ and 1 .

$I: \sigma=0,5 ; 2: \sigma=0,75: 3: \sigma=1$ (valeur de $k$ selon la figure 1 pour un ciel standard).

$$
\begin{aligned}
& \mathrm{R}=0,25 \text { pour } \mathrm{DO}=5,98 \text { si } \sigma=0,5 \quad \mathrm{~F}=9,8 \\
& \mathrm{DO}=3,82 \quad \text { si } \quad \sigma=0,75 \quad \mathrm{~F}=5,9 \\
& \mathrm{DO}=2,73 \quad \text { si } \quad \sigma=1 \quad \mathrm{~F}=4,1
\end{aligned}
$$


Ces peuplements situés autour du village d'Otawa, dans la région de Taï, âgés de 4 à 55 ans (colonne 1, tableau II), peuvent être considérés comme caractéristiques de la reconstitution forestière après abandon de champs sur brûlis, d'après F. KAHN qui les a étudiés sur le plan structural (1981).

Le ciel pendant la journée de mesure a été changeant, de nuageux à très couvert avec averses, conditions peu propices au type de mesures projetées.

Compte tenu du temps disponible, il n'a pas été possible de faire un échantillonnage systématique et on s'est basé sur une exploration à l'œil des différentes structures rencontrées dans chaque station. Pour chaque station, le nombre de mesures (n) est indiqué (colonne 2) sur le tableau II, ci-dessous, où on a rappelé les mesures faites sous forêt dense (apparemment vierge).

Nous avons porté les différentes valeurs du rapport $R$ et celles de l'EDR sur la figure 4 (en logarithme) et sur le tableau II (colonnes 3 et 5 ).

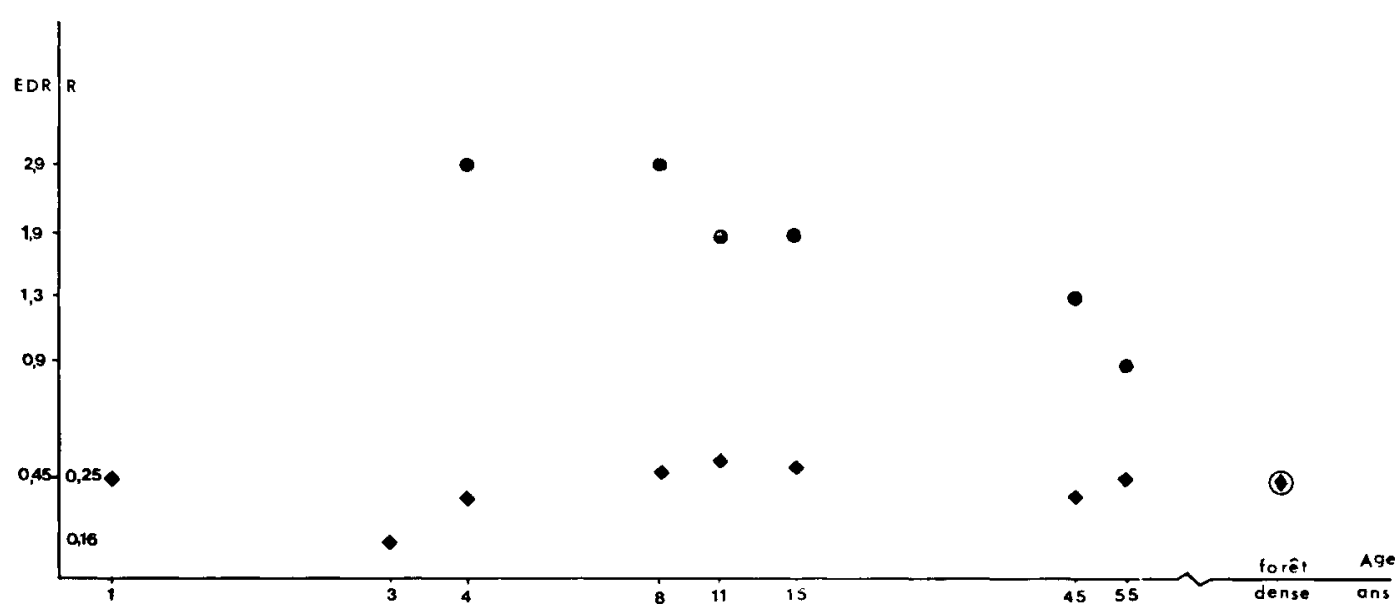

FIG. 4

Evolution de l'éclairement diffus relatif EDR et du rapport spectral $R$ sous des peuplements cicatriciels après culture (échelles logarithmiques).

Evolution of the diffuse site factor $(E D R)$ and of the spectral ratio $R$ under secondary stands (log. scales).

On constate que le rapport spectral $R$ défini plus haut $\left(R=\frac{i_{1} \times i_{02}}{i_{01} \times i_{2}}\right)$

(colonne 3) est pratiquement constant (") tandis que les valeurs de l'EDR diminuent lentement (colonne 5) au cours de l'évolution du peuplement des friches.

(*) Stoutjesdisk (1972) trouve à Java un rapport visible/infra-rouge plus petit sous une forêt secondaire que sous une forêt vierge. 
L'écart-type (colonne 4) des valeurs de $\mathrm{R}$ traduit bien évidemment l'homogénéité ou l'hétérogénéité structurale des peuplements. Il est faible pour la station âgée de 4 ans qui possède un beau peuplement homogène de Macaranga hurifolia (cf. KAHN \& DE NAMUR, 1978). Au moment du dépérissement du Macaranga ( 8 ans), il est très élevé, ce qui reflète bien l'hétérogénéité de ce stade et il diminue ensuite progressivement, parallèlement à la reconstitution d'un couvert continu régulier.

La constance du rapport $R$ tandis que l'EDR diminue est en contradiction avec les mesures précédentes et la théorie immédiate si l'on se réfère à l'équation (10). Avant de chercher ailleurs une explication à ce phénomène, il faut écarter l'éventualité d'une simple erreur grossière.

Une certaine augmentation de l'indice foliaire (même faible) au cours de l'évolution des peuplements cicatriciels, avec une baisse concomitante de l'éclairement diffus relatif au sol semblant aller de soi, on ne peut donc mettre en question que la réalité de la stabilité du rapport spectral $\mathrm{R}$. Or nous avons fait quelques mesures autour d'Adiopodoumé (région d'Abidjan) sur des peuplements jeunes ( 1 à 3 ans) et constaté, dès l'âge de 1 an, des valeurs analogues aux valeurs observées sur des peuplements plus âgés et même un enrichissement relatif en infra-rouge plus grand, puisquion a mesuré, sous un peuplement de 3 ans à Eupatorium odoratum (*), sous-ligneux à port lianescent, un rapport $R$ de 0,16 (cf. fig. 4). Ces observations permettent de confirmer la réalité du phénomène dont nous devons donc chercher une explication.

Reprenons l'équation simplifiée (6'), après transformation logarithmique :

$$
-\log \left(i / i_{0} \lambda\right)=\left(1-1,4 \sigma_{(\theta, \ldots)} T \lambda\right) K_{(F, \theta)} F \text { (densité optique) }
$$

où les indices $i, \lambda$ et $F$ indiquent que le coefficient est fonction de la structure $(\theta)$, de la longueur d'onde $(\lambda)$ et de l'indice foliaire $(F)$.

Si l'on fait le rapport entre la densité optique (DO $=-\log E D R)$ et le Logarithme du rapport spectral, il vient :

$$
\mathrm{DO} / \log \mathrm{R}=\left(1-1,4 \sigma_{(\mathrm{i})} \text { Tvis }\right) / 1,4 \sigma_{(i)} \text { (Tvis - Tglobal) }
$$

qui montre que la seule source théorique de variation du rapport $\mathrm{DO} / \log \mathrm{R}$ est une variations de $\sigma_{(\mathrm{i})}$.

Cette évolution du coefficient de dispersion $\sigma$ peut avoir une explication simple. La voûte forestière fait écran à la pénétration de la lumière à la fois par ses feuilles et par ses branches. Or si les feuilles sont transparentes à l'infra-rouge, les branches sont, elles, opaques. Si l'on tient compte du fait qu'une partie seulement de l'indice «foliaire» total est constitué par des feuilles susceptibles de transmettre ou de réfléchir la lumière, l'autre partie étant constituée par des organes opaques (noir), on obtient une formulation où le coefficient de dispersion $\sigma$ doit inclure la part

(*) Ces peuplements à Eupatorium, et les peuplements héliophiles jeunes d'une façon générale, tels que nous les avons observés, sont remarquables par un port fortement planophile et surtout par un non recouvrement des feuilles. Dès que les feuilles supérieures ombragent les feuilles inférieures, ces dernières meurent et tombent. Au total, les propriétés optiques de ces couverts peuvent se rapprocher de celles d'une feuille unique et continue et auraient donc un rapport spectral limite $R_{0}=T$ (vis) $/ T($ vis $+I R)=0,11$. 
relative des feuilles dans l'indice foliaire apparent. Or, avec l'évolution de la végétation, on sait que les organes non assimilateurs prennent une part croissante (augmentation de la biomasse sans augmentation sensible de l'indice foliaire (cf. EWEL, 1971). Ceci justifie d'une diminution dans le temps de l'indice $\sigma$ et explique, partiellement au moins, le phénomène observé.

On peut écrire $\log \mathrm{R}$ à partir de $\left(6^{\prime}\right)$, il vient :

$$
\log \mathrm{R}=1,4 \sigma \mathrm{KF}\left(\mathrm{T}_{1}-\mathrm{T}_{2}\right)
$$

Cette équation montre que la valeur d'équilibre de $\mathrm{R}=0,25$ s'obtient pour tout :

$$
\begin{aligned}
\operatorname{Ln} 0,25= & \sigma \mathrm{KF} \cdot 1,4(0,0423-0,3788) \\
& \sigma \mathrm{KF}=2,94 \simeq 3
\end{aligned}
$$

Pour une forêt dense, avec la valeur de $\sigma=1 / 2$, admise au début de ce texte, il viendrait $\mathrm{KF}=6$, ce qui correspond si lon se reporte à la fig. 1 à $\mathrm{K}=0,62$ et $\mathbf{F}=9,75$ et $\mathrm{EDR}=0,25$ p. 100 (l'EDR mesuré, qui correspond à $\mathrm{KF}=5,4$, est de 0,45 p. 100). Pour un couvert héliophile jeune, si on admet $\sigma=k=1$, il vient : $\mathrm{KF}=3, \mathrm{EDR}=5$ p. 100 et $\mathrm{F}=3$.

D’une manière générale, on peut dériver $\sigma$ de (5) et (11) :

$$
\sigma=-0,96 \mathrm{Ln} \mathrm{R} / 0,47 \mathrm{LnEDR}=-2,04 \mathrm{Ln} \mathrm{R} / \mathrm{Ln} \mathrm{EDR}
$$

valeurs portées sur le tableau II, colonne 8.

\begin{tabular}{|c|c|c|c|c|c|c|c|}
\hline $\begin{array}{c}1 \\
\text { Age }\end{array}$ & $\begin{array}{l}2 \\
n\end{array}$ & $\begin{array}{l}3 \\
\mathrm{R}\end{array}$ & $\begin{array}{c}4 \\
\mathrm{~cd}\end{array}$ & $\begin{array}{c}5 \\
\text { EDR } \%\end{array}$ & $\stackrel{6}{\sigma \mathrm{KF}}$ & $\begin{array}{c}7 \\
\text { KF }\end{array}$ & $\begin{array}{l}8 \\
0\end{array}$ \\
\hline 4 & 16 & 0215 & 0.03 & 2.9 & 3,34 & 3,65 & 093 \\
\hline 8 & 24 & 0,260 & 021 & 29 & 2,93 & 3,65 & 0.80 \\
\hline 11 & 24 & 0.275 & 018 & 1,9 & 2,81 & 4.08 & 0,69 \\
\hline 15 & 26 & 0265 & 0,12 & 1,9 & 2,89 & 4.08 & 0,71 \\
\hline 45 & 37 & () 220 & 0,11 & 1.3 & 3,29 & 4,47 & 0,74 \\
\hline 55 & 26 & 0250 & 011 & 09 & 3,01 & 4.85 & 0,62 \\
\hline $\begin{array}{l}\text { Foret } \\
\text { dense }\end{array}$ & & 0250 & 010 & () 45 & 3.01 & 5.56 & 0.54 \\
\hline
\end{tabular}

\section{TABleau 2}

Caractéristiques lumineuses de couverts en cours d'évolution.

Evolution of light characteristics during succession.

\section{Conséquence de l'évolution de $R$}

La précocité de la dérive spectrale a d'importantes conséquences écologiques. On sait en effet depuis les travaux de Meıschke (1936) que la germination de très nombreuses espèces est inhibée par la lumière riche en infra-rouge qui est transmise 
par les feuilles. Il est apparu depuis que c'est le rapport des longueurs d'onde 660 et $730 \mathrm{~nm}$ qui jouc par l'intermédiaire des phytochromes ce rôle essentiel sur la germination des espèces à dormance photolabile.

Les graines de ces espèces restent dormantes dans un sol couvert de végétation et ne germent que si le couvert est détruit, ce qui leur assure du même coup une lumière suffisante pour leur croissance. La vitesse de germination de ces graines varie beaucoup selon l'espèce, et si certaines espèces ont une germination très rapide, des Mélastomacées de Guyane germent encore au bout de 5 mois (PrEvost, 1980).

La première vague de végétation qui s'installe sur le sol dénudé produit rapidement des conditions spectrales telles que parmi les espèces à dormance photolabile, celles dont la germination est très lente sont inhibées. Aussi un important stock édaphique de semences herbacées à germination rapide entrave l'expression du potentiel des arbustives à germination plus lente si des désherbages précoces ñinterviennent pas. C'est ce que nous avons observé au cours d'une étude de l'écologie de Trema guineensis (Alexandre, 1978) et qui explique sans doute que des cycles culturaux à jachère trop courte conduisent à la disparition des recrûs arbustifs qui sont progressivement remplacés par des formations herbacées qui deviennent plus ou moins stables sous l'effet dı feu.

\subsection{Vérification du bon emp!oi du luxmètre pour la mesure de l'éclairement diffus relatif}

Le luxmètre au sélénium est un appareil qui a été beaucoup critiqué pour les mesures écologiques concernant la croissance des plantes, en raison de sa réponse spectrale imitant celle de l'œil humain, avec un pic de sensibilité relativement étroit dans le jaune. Ce pic dans le jaune se rapproche du pic relatif de transmission des feuilles dans le vert. De plus, lappareil est peu sensible dans le rouge, couleur essentielle pour la photosynthèse, mais en revanche garde une (très) légère réponse dans le très proche infra-rouge (ou rouge sombre) qui n'est pratiquement pas absorbé par les végétaux. Cet ensemble de caractères fonde les critiques usuelles.

Ainsi que nous l'avons déjà mentionné, nous avons mesuré, selon un échantillonnage systématique, l'éclairement diffus relatif du sous-bois de la forêt de Taï à l'aide d'un appareil conçu tout exprès, le quantomètre Lambda. L'EDR moyen ainsi obtenu est de 0,45 p. 100 . La même mesure au luxmètre conne une moyenne de 0,7 p. 100. Lécart est de 56 p. 100 , ce qui cst considérable. Le coefficient de transmission des feuilles mesuré au luxmètre $(5,5$ p. 100) permet de calculer un EDR théorique :

$$
\mathrm{EDR}=\mathrm{e}^{-\mathrm{kF}(1-\mathrm{T} / 2)}=0,00463 \quad(\text { avec } \mathrm{kF}=5,52)
$$

soit un écart, par rapport au quantomètre, inférieur à 3 p. 100, ce qui est peu et serait le plus souvent négligeable, et permettrait d’utiliser valablement le luxmètre.

Ce n'est donc pas la réponse spectrale du luxmètre qui explique le fait qu’il donne une mesure surévaluée par rapport à un type d'appareil spécialement adapté.

Ainsi quil a été montré par ANDERSON ou Cowan (cf. supra), la transparence d'un couvert diminue avec langle d'incidence. En sous-bois, on rencontre un 
rayonnement dont la composante verticale est prépondérante, et la transparence est voisine de zéro pour des incidences supérieures à 70 (MADGWICK et al., 1969). En revanche, lors de la mesure de référence à découvert, la composante de forte incidence n'est pas négligeable, puisque, par définition même de l'Eclairement Diffus Relatif, la mesure est faite par ciel couvert.

Or, le luxmètre utilisé, comme beaucoup de luxmètres, contrairement au capteur PAR Lambda, est dépourvu de dispositif «correcteur de cosinus». Il s'ensuit que ce luxmètre sous-évalue l'éclairement à découvert beaucoup plus que l'éclairement sous couvert, d'où un rapport d'EDR surévalué. Un simple globe en verre dépoli constitue un grossier «correcteur de cosinus» qui nous a permis d'estimer l'erreur due aux rayons obliques à quelque 16 p. 100 à midi par temps couvert et jusqu'à 69 p. 100 au coucher du soleil. Or c'est peu avant le coucher du soleil quavaient été faites les mesures d'EDR au luxmètre.

A condition de tenir compte de cette cause d'erreur, le luxmètre pourrait donc redevenir un excellent instrument pour l'écologiste en raison de sa grande surface captrice permettant de diminuer le nombre de mesures, de sa solidité, de sa grande sensibilité, enfin de son prix peu élevé comparé à celui d'un quantomètre.

\section{Conclusion}

Les valeurs que permet de calculer notre modèle établi pour décrire un couvert «sphérique» et celles que nous avons mesurées dans le sous-bois de la forêt de Taï dans deux bandes spectrales $(0,4$ à 0,7 et 0,4 à $1,1 \mu)$ sont très voisines. On peut donc conclure que la structure du couvert de cette forêt est de type sphérique. Cependant d'autres méthodes d'étude permettent de nuancer cette approximation (ALEXANDRE, 1979 a).

Il est à remarquer que notre modèle attribue à la dispersion par les feuilles une part dans l'éclairement du sous-bois plus faible que les modèles habituellement utilisés. Il explique bien la part relative de l'infra-rouge proche dans la pénombre du sous-bois qui, tout en étant élevée, n'atteint pas les valeurs que laissent prévoir d'autres modèles. Mais, fait essentiel, nous avons été amené à mettre en évidence que, très tôt, au cours de la colonisation d'un sol nu, l'enrichissement en infra-rouge de la lumière transmise par le couvert des pionnières est égal, voire supérieur, à celui observé sous des couverts évolués. Cette rapidité de la modification spectrale a d'importantes conséquences écologiques car la germination d'un grand nombre d'espèces, surtout pionnières, est inhibée par une lumière riche en infra-rouge proche.

Reçu pour publication en décembre 1981.

\section{Remerciements}

L'auteur tient à remercier M. Alain Perrier pour toutes ses remarques bienveillantes. 


\section{Summary \\ Light penetration to the undergrowth of a tropical rain forest}

A simple stochastic model of diffuse light penetration, where leave transmission and reflection factors are considered, is proposed for large LAI stands with spherical leave distribution.

Mean values of undergrowth lighting in Taï Tropical Rain Forest, measured in two contrasting wave-bands well agree with the model. Then we show that, in the undergrowth, the light spectral ratio, in the same two wave-bands, is almost constant regarding to stand evolution after clearing; therefore such ratio cannot be used for the determination of Leaf Area Index. This seemingly unconsistency can in fact be accounted by the model when considering a dispersion coefficient that varies according to the relative proportion of branches and leaves.

We discuss selenium cell utilization and we find that it may be usable for most ecological research under tropical rain forest regardless of its known poor spectral response as long as the cosine factor is corrected.

$$
\text { Key-words : Model - Lighting - Forest - Ivory Coast }
$$

\section{Références bibliographiques}

AleXandre D.-Y., 1978. Observations sur l'écologie de Trema guineensis en Basse Côted'Ivoire. Cah. O.R.S.T.O.M., sér. Biol., 13 (3), 261-266.

Alexandre D.-Y., 1979 a. Etude des conditions lumineuses au niveau du sous-bois de la forêt de Taï. A paraître in Ecol. gen.

ALeXANDRE D.-Y., 1979 b. L'indice foliaire des forêts tropicales : analyse bibliographique. A paraître in Ecol. gen.

AleXandRe D.-Y., 1979 c. De la régénération naturelle à la sylviculture en forêt tropicale. Multigraphie O.R.S.T.O.M., 33 p.

Alexandre D.-.Y, 1979 d. Aspects de la régénération naturelle en forêt dense de Côted'Ivoire. Multigraphie O.R.S.T.O.M., 9 p.

ANDERson M.C., 1966. Stand structure and light penetration. II - A theoretical analysis. J. appl. Ecol., 3, 41-54.

Anderson M.C., 1969. A comparison of two theories of scatering of radiation in crops. Agric. Meteorol., 6, 399-405.

Atkins W.R.G., Poole H.H. et Stanbury F.A., 1937. The measurement of the intensity and the colour of the light in woods by means of emission and rectifier photoelectric cells. Proc. r. Soc. London, ser. B, 121, 427-450.

Bonhomme R. et Varlet Grancher C., 1977. Application aux couverts végétaux des lois de rayonnement en milieu diffusant. I - Etablissement des lois et vérification expérimentale. Ann. Agron., 38 (6), 567-582.

Chartier Ph., 1966. - Etude du microclimat lumineux dans la végétation. Ann. Agron., 17 (5), 571-602.

Chartier Ph. et al., 1973. Effets physiologiques et caractérisation du rayonnement solaire dans le cadre d'une méthode d'aménagement sylvicole en forêt dense africaine. Bois For. Trop., $\mathrm{n}^{0} 152,19-35$.

Cowan I.R., 1968. The interception and absorption of radiation in plant stands. J. appl. Ecol., 5, 367-379. 
EwEL J., 1971. Biomass changes in early tropical succession. Turrialba, 21 (1), 110-112.

GaUSMAN et al., 1970. Reflectance transmittance and absorptance of light of leaves for 11 plant genera with different leaf mesophyll arrangements. Texas $\mathrm{A}$ and $\mathrm{M}$ University. Technical monograph, $\mathrm{n}^{\circ} 7,38 \mathrm{p}$.

Huttel C., 1975. Recherches sur l'écosystème de la forêt subéquatoriale de Basse Côted'Ivoire. Chap. 3 : Inventaire et structure de la végétation ligneuse. La Terre et la Vie, 29 (2), 178-191.

KaHN F., 1981. La reconstitution de la forêt tropicale après culture traditionnelle (Sud-Ouest de la Côte-d'Ivoire). Thèse $3^{0}$ cycle Orsay, $176 \mathrm{p}$.

KAHN F., DE Namur Ch., 1978. Le stade à Macaranga hurifolia : installation, développement et disparition. Cah. O.R.S.T.O.M., sér. Biol., 13 (3), 255-260.

Kasanaga M., Monsi M., 1954. On the light transmission of leaves and its meaning for production of matter in plant communities. Jpn. J. Bot., 14, 304-324.

Lemeur R., BLad B.L., 1974. A critical review of light models for estimating the short wave radiation regime of plant canopies. Agric. Meteorol., 14, 255-286.

Madgwick H.A.I., Brumfield G.L., 1969. The use of hemisperical photographs to assess light climate in forest. J. Ecol., 57 (2), 537-542.

MeischKe D., 1936. Uber den Einfluss des Strahlung auf Licht- und Dunkelkeimer. Jahrb. Wissensch. Bot., 83, 359.

Monteith J.L., 1965. Light distribution and photosynthesis in fields crops. Ann. Bot., 29, 17-37.

NiLson T., 1971. A theoretical analysis of the frequency of gaps in plant stands. Agric. Meteorol., 8, 25-38.

Odum H.T., Copeland B.J., Brown R.Z, 1963. Direct and optical assay of leaf mass of the lower montane rain forest of Puerto Rico. Proc. natl Acad. Sci. U.S.A., 49, 429-434.

Prevost M.F., 1980. Mise en évidence de graines d'espèces pionnières dans le sol de la forêt primaire de Guyane. Proposé à Turrialba.

StoutjesdiJK P., 1972. A note on the spectral transmission of light by tropical rain forest. Acta bot. neerl., 21, 346-350.

SzEICZ G., 1974. Solar radiation in crop canopies. J. appl. Ecol., 11, 1117-1156.

YoDA K., 1974. Three dimensional distribution of light intensity in a tropical rain forest of West Malaya. Jap. J. Ecol., 24 (4), 247-254. 
ANNEXES 1 et 2
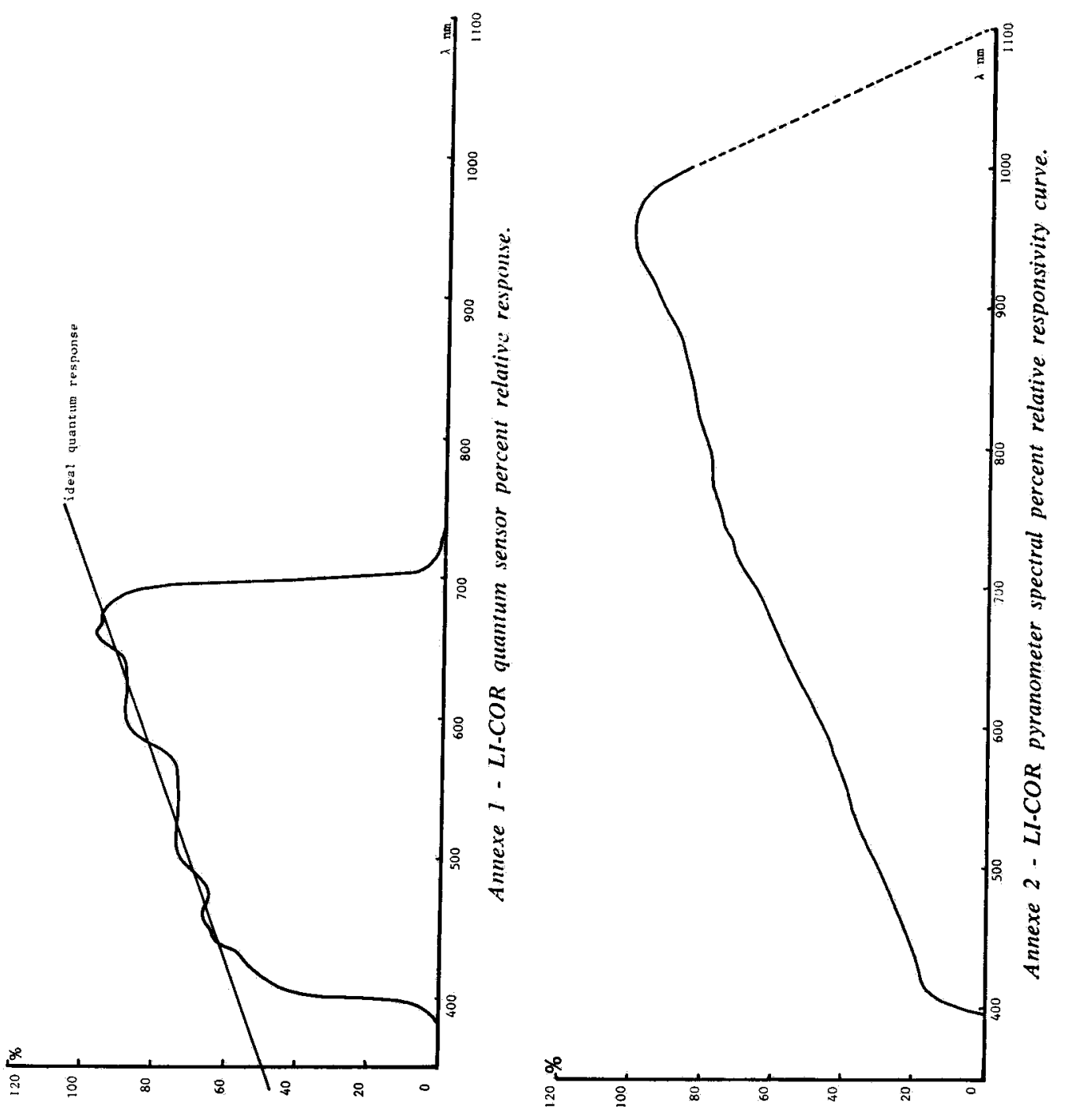